
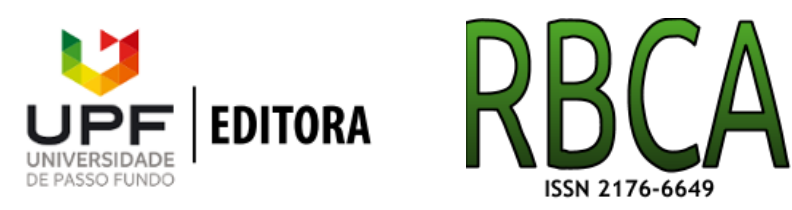

Revista Brasileira de Computação Aplicada, Novembro, 2021

DOI: 10.5335/rbca.v13i3.12653

Vol. 13, № 3, pp. 86-100

Homepage: seer.upf.br/index.php/rbca/index

ARTIGO ORIGINAL

\title{
AutoRL-TSP-RSM: sistema de aprendizado por reforço automatizado com metodologia de superfície de resposta para o problema do caixeiro viajante
}

\section{AutoRL-TSP-RSM: automated reinforcement learning system with response surface methodology for the traveling salesman problem}

\author{
Gleice Kelly Barbosa Souza ${ }^{1}$ and André Luiz Carvalho Ottoni ${ }^{(\mathbb{D}, 1}$ \\ ${ }^{1}$ Centro de Ciências Exatas e Tecnológicas (CETEC), Universidade Federal do Recôncavo da Bahia (UFRB) \\ *kelly.189@hotmail.com; andre.ottoni@ufrb.edu.br
}

Recebido: 11/06/2021. Revisado: 17/11/2021. Aceito: 29/11/2021.

\section{Resumo}

A definição de parâmetros é uma importante etapa para a utilização de métodos de Aprendizado de Máquina. No entanto, pode ser altamente custoso definir esses valores de condições iniciais para cada aplicação. Assim, este trabalho tem como objetivo propor um sistema de Aprendizado de Máquina Automatizado para ajuste de parâmetros. Nesta linha, foi desenvolvido um método de Aprendizado por Reforço Automatizado aplicado ao Problema do Caixeiro Viajante. O sistema proposto ajustou através da Metodologia de Superfície de Resposta dois parâmetros (taxa de aprendizado e fator de desconto) do algoritmo Q-learning. Os resultados revelaram que os valores ajustados pelo método proposto alcançaram, em geral, as melhores soluções, em comparação com a adoção de parâmetros da literatura.

Palavras-Chave: Aprendizado por Reforço; AutoML; Problema do Caixeiro Viajante.

\section{Abstract}

The tuning of parameters is an important step towards the use of machine learning methods. However, it can be costly to define these initial condition values for each application. Thus, this paper aims to propose an Automated Machine Learning system for parameter tuning. In this line, an Automated Reinforcement Learning method was developed applied to the Traveling Salesman Problem. The proposed system adjusted through the Response Surface Methodology two parameters (learning rate and discount factor) of the Q-learning algorithm. The results revealed that the values adjusted by the proposed method reached, in general, the best solutions, in comparison with the adoption of parameters from the literature.

Keywords: AutoML; Reinforcement Learning; Traveling Salesman Problem.

\section{Introdução}

O Aprendizado de Máquina, em inglês, Machine Learning (ML), é uma área multidisciplinar que envolve temas como 
Inteligência Artificial (IA), probabilidade, estatística, complexidade computacional e psicologia (Mitchell, 1997, Sutton and Barto, 2018). Em ML, o objetivo é o desenvolvimento de sistemas capazes de aprender com suas experiências, de forma a melhorar no desempenho da execução da tarefa que foi designada (Monard and Baranauskas, 2003, Celiberto Jr, 2007, Stange, 2011, Russell and Norvig, 2013). Nesse aspecto, técnicas de ML podem ser aplicadas em diversos contextos, como: classificação, análise de dados, robótica, jogos e reconhecimento de padrões (Mitchell, 1997, Bianchi, 2004, Serra, 2004, Rossi, 2015, Sutton and Barto, 2018, Boeing et al., 2019, Alzubaidi et al., 2021).

Uma dos grandes desafios para o bom desempenho na utilização de métodos de aprendizado de máquina é a definição de quais algoritmos e parâmetros serão utilizados durante os experimentos (Brazdil et al., 2008, Hutter et al., 2018, Tuggener et al., 2019). A complexidade desta etapa pode ser explicada pelo fato que para cada situação pode existir uma configuração específica de algoritmo e/ou parâmetro que irá fornecer melhores resultados (Feurer et al., 2015, Makmal et al., 2016, Mantovani et al., 2019, Cai et al., 2020). Assim, diversos métodos já foram propostos na literatura para o ajuste dessas configurações iniciais, dentre elas têm-se: Metodologia de Superfície de Resposta (RSM - Response Surface Methodology) (Ottoni, Nepomuceno and Oliveira, 2016, Ottoni et al., 2019, Lakshmi et al., 2020), Simulação Projetiva (Makmal et al., 2016), Métodos Empíricos (Gershman, 2016), Algoritmos Bioinspirados (Rossi, 2009), Algoritmo Firefly (Wang et al., 2017) e Algoritmos Genéticos (Espindola, 2009, Feitosa et al., 2009, Zhao et al., 2012).

Nesse sentido, de forma a automatizar o processo de seleção parâmetros e algoritmos de $M L$ surgiu o Automated Machine Learning (AutoML). Os sistemas de AutoML, podem ser utilizados sob duas vertentes, a da recomendação (Brazdil et al., 2008, Mantovani et al., 2015, Cai et al., 2020) e a da otimização (Hutter et al., 2018, Tsiakmaki et al., 2019, Stamoulis et al., 2020). Uma das principais contribuições dos sistemas de AutoML encontra-se na redução do esforço empregado pelo experimentador para definir as condições adequadas para os métodos de ML, dado que, tais configurações são definidas automaticamente pelo sistema (Brazdil et al., 2008, Hutter et al., 2018).

Uma possível área de aplicação de AutoML é na definição de condições experimentais de Aprendizado por Reforço (RL) (Ottoni, Ottoni, Oliveira and Nepomuceno, 2020). No $\mathrm{RL}, \mathrm{o}$ agente aprende a partir de interações com o ambiente, de forma a buscar as decisões que proporcionem os maiores retornos (Martins, 2007, Sutton and Barto, 2018, Bianchi, 2004, Serra, 2004, Santos, 2009, Santos et al., 2014, Sutton and Barto, 2018). Ao tomar essas decisões, o agente irá receber reforços (recompensa ou punição) que irão determinar a natureza da decisão tomada (Martins, 2007, Russell and Norvig, 2013, Santos et al., 2014). O RL pode ser aplicado nas mais diversas situações, dentre elas: jogos (Russell and Norvig, 2013, Sutton and Barto, 2018), robótica (Bianchi, 2004, Goldbarg and Luna, 2005, Martins, 2007, Russell and Norvig, 2013, Ottoni, 2016, Ottoni, Nepomuceno and Oliveira, 2016, Sutton and Barto, 2018), sistemas multiagentes (Ottoni, 2016, Ottoni, Nepomuceno and Oliveira, 2016). Outra importante área de aplicação de Aprendizado por Reforço é na otimização combinató- ria (Santos, 2009, Ottoni et al., 2017, Alipour et al., 2018, Ottoni, Nepomuceno, de Oliveira and de Oliveira, 2020).

Nesse aspecto, trabalhos recentes na literatura apresentaram metodologias para ajuste de parâmetros de Aprendizado por Reforço aplicado em um conhecido problema de otimização combinatória, o Problema do Caixeiro Viajante (PCV) (Ottoni et al., 2018, Ottoni, Ottoni, Oliveira and Nepomuceno, 2020). Ottoni et al. (2018) utilizam a Metodologia de Superfície de Resposta (RSM) para realizar a definição de dois parâmetros (taxa de aprendizado e fator de desconto) de RL aplicado no PCV. No entanto, em Ottoni et al. (2018) os parâmetros não foram gerados como um processo de AutoML. Por outro lado, Ottoni, Ottoni, Oliveira and Nepomuceno (2020) propõem um sistema de Aprendizado por Reforço automatizado (AutoRL) para o Problema do Caixeiro Viajante utilizando o método Variable Neighborhood Search. Dessa forma, o presente estudo busca avançar esses estudos recentes da literatura, automatizando o processo de geração de parâmetros com RSM.

Baseando-se na relevância da aplicação do Aprendizado por Reforço em problemas de otimização combinatória, como Problema do Caixeiro Viajante (Gambardella and Dorigo, 1995), Problema da Mochila (Ottoni et al., 2017) e Sequential Ordering Problem (Ottoni, Nepomuceno, de Oliveira and de Oliveira, 2020), o objetivo deste trabalho é propor um sistema de AutoRL aplicado ao Problema do Caixeiro Viajante. Para isso, será automatizado o processo de ajuste de parâmetros com Metodologia de Superfície de Resposta, utilizando a modelagem proposta por Ottoni et al. (2018).

Este artigo está divido em seções. A seção 2 demonstra conceitos teóricos. A seção 3, apresenta a metodologia empregada durante a construção deste trabalho. Já na seção 4, são apresentados os resultados. Por fim, na seção 5 é apresentada a conclusão.

\section{Fundamentação Teórica}

\subsection{Aprendizado de Máquina}

O Aprendizado de Máquina (ML) é um campo da Inteligência Artificial (IA) que pode ser dividido em três categorias (Russell and Norvig, 2013):

- Aprendizado Supervisionado: no aprendizado supervisionado, o agente recebe um conjunto de entradas e saídas que servem como um professor para lhe instruir sobre qual é a saída esperada para cada entrada (Russell and Norvig, 2013, Ottoni, 2016).

- Aprendizado por Reforço: no aprendizado por reforço, o agente não recebe informações prévias sobre $\mathrm{o}$ ambiente, ele deve aprender a medida que interage com o ambiente e os reforços recebidos devem definir quais serão suas próximas decisões (Martins, 2007, Russell and Norvig, 2013, Ottoni, 2016).

- Aprendizado não Supervisionado: no aprendizado não supervisionado, o agente recebe os dados de entrada, mas não recebe exemplos de como devem ser os dados de saída. $O$ próprio agente deve realizar o agrupamento de dados que sejam semelhantes e que possivelmente pertençam a um mesmo grupo (Russell and Norvig, 2013). 


\subsubsection{Aprendizado por Reforço}

Aprendizado por Reforço (RL) é uma das áreas que envolve IA e seu funcionamento se baseia em tentativas e erros (Santos et al., 2014). No RL, o agente realiza uma ação em um determinado estado e em seguida recebe um reforço indicando seu sucesso ou fracasso (Mitchell, 1997, Faria, 2000, Bianchi, 2004). Ao realizar estas ações, o agente interage com o ambiente e aprende sobre onde se encontra. Assim, o mesmo busca realizar ações que vão lhe render as melhores recompensas, independentemente destas serem a curto ou a longo prazo (Russell and Norvig, 2013, Ottoni, 2016, Sutton and Barto, 2018).

\subsubsection{Processos de Decisão de Markov}

Os Processos de Decisão de Markov (Markov Decision Process - MDP) são utilizados para tomadas de decisões sobre situações incertas (Pellegrini and Wainer, 2007). O RL, tem em sua base o MDP, dado que, no RL o agente não sabe qual resultado sua ação irá gerar, sabendo somente após a realização da ação (Sutton and Barto, 2018).

Os MDP, possuem um conjunto de variáveis para o controle do ambiente, dentre elas: ação, estado, recompensa e uma função probabilística para transição de estado (Pellegrini and Wainer, 2007). Estas variáveis podem ser representadas pela quádrupla $(S, A, T, R)$ (Pellegrini and Wainer, 2007, Ottoni, 2016), sendo:

- S o conjunto de estados;

- A o conjunto de ações;

- T a função probabilística;

- $R$ a recompensa.

\subsubsection{Algoritmos de Aprendizado por Reforço}

Diversos algoritmos podem ser aplicados ao RL, alguns deles são o método de Diferença Temporal, o Q-learning e o Sarsa. Sendo que, os dois últimos são baseados no método de Diferença Temporal (Ottoni, 2016, Sutton and Barto, 2018).

\subsubsection{Diferença Temporal.}

O método de Diferença Temporal utiliza a diferença de utilidades entre estados consecutivos (Russell and Norvig, 2013). Este método foi proposto por Sutton (1988) e sua função é apresentada a seguir (Russell and Norvig, 2013, Sutton and Barto, 2018):

$$
U(s)=U(s)+\alpha\left[R(s)+\gamma U\left(s^{\prime}\right)-U(s)\right]
$$

em que,

$$
\begin{aligned}
& \text { - U(s) é a utilidade no estado atual; } \\
& \text { - } \alpha \text { é a taxa de aprendizado; } \\
& \text { - } \gamma \text { é o fator de desconto; } \\
& \text { - } R(\text { (s) é a recompensa; } \\
& \text { - } U\left(s^{\prime}\right) \text { é a utilidade no estado futuro. }
\end{aligned}
$$

\subsubsection{Q-learning.}

OQ-learning, é baseado no método de Diferença Temporal para situações em que não há modelo (Watkins and Dayan, 1992, Sutton and Barto, 2018). O Q-learning foi proposto por Watkins (1989) e sua função é exibida a seguir (Watkins and Dayan, 1992, Sutton and Barto, 2018):

$$
Q_{t+1}\left(s_{t}, a_{t}\right)=Q_{t}\left(s_{t}, a_{t}\right)+\alpha\left[r\left(s_{t}, a_{t}\right)+\gamma \max _{a^{\prime}} Q\left(s^{\prime}, a^{\prime}\right)-Q_{t}\left(s_{t}, a_{t}\right)\right]
$$

em que,

- Q é a matriz de aprendizado;

- $r(s, a)$ é o reforço recebido por realizar a ação $a_{t}$ no estado $s_{t} ;$

- $\alpha$ é a taxa de aprendizado;

- $\gamma$ é o fator de desconto;

- $s_{t}$ é o estado atual;

- $a_{t}$ é a ação realizada no estado atual;

- s'é o estado futuro;

- $a^{\prime}$ é a ação que será realizada no estado futuro.

\subsubsection{Sarsa.}

O Sarsa é uma modificação do Q-learning e seu nome foi dado por Sutton (1996). A modificação realizada no Qlearning se deu na forma em que a recompensa futura é concebida, enquanto o Q-learning recebe a recompensa máxima o Sarsa recebe a recompensa esperada (Sutton and Barto, 2018). Assim, sua equação é:

$$
Q_{t+1}\left(s_{t}, a_{t}\right)=Q_{t}\left(s_{t}, a_{t}\right)+\alpha\left[r\left(s_{t}, a_{t}\right)+\gamma Q\left(s^{\prime}, a^{\prime}\right)-Q_{t}\left(s_{t}, a_{t}\right)\right]
$$

em que,

- $Q$ é a matriz de aprendizado;

- $r(s, a)$ é o reforço recebido por realizar a ação $a_{t}$ no estado $s_{t} ;$

- $\alpha$ é a taxa de aprendizado;

- $\gamma$ é o fator de desconto;

- $s_{t}$ é o estado atual;

- $a_{t}$ é a ação realizada no estado atual;

- s'é o estado futuro;

- $a^{\prime}$ é a ação que será realizada no estado futuro.

\subsubsection{Parâmetros}

- Taxa de aprendizado: a taxa de aprendizado $(\alpha)$ controla a velocidade com que o agente aprende. Este parâmetro pode variar entre o e 1 . Sendo que, para os casos em que $\alpha=0$ não existe aprendizado (Ottoni, Nepomuceno and Oliveira, 2016).

- Fator de desconto: o fator de desconto $(\gamma)$ determina o quão importante é uma recompensa recebida pelo agente. Este parâmetro pode variar entre o e 1. Sendo que, para valores de $\gamma$ próximos de 0 , as recompensas são insignificantes. Em contrapartida, quanto mais próximo de 1 for o valor de $\gamma$, mais significativa será a recompensa (Celiberto Jr, 2007, Martins, 2007, Russell and Norvig, 2013, Ottoni, 2016).

- Política $\epsilon$-greedy: a política $\epsilon$-greedy $(\epsilon)$ determina o quão aleatórias são as decisões tomadas pelo agente. Assim como os parâmetros apresentados anteriormente, o valor da política $\epsilon$-greedy também pode variar entre o e 1 (Celiberto Jr, 2007, Martins, 2007, Ottoni, 2016). Como regra de atualização da política $\epsilon$-greedy tem-se (Celiberto Jr, 2007, Ottoni, 2016):

$$
\pi(s)=\left\{\begin{array}{l}
a^{*}, \text { com probabilidade } 1-\epsilon \\
a_{a}, \text { com probabilidade } \epsilon
\end{array}\right.
$$


em que,

- $\pi(s)$ é a política para o estado atual;

- $a^{*}$ é a melhor ação disponível para o estado atual;

- $a_{a}$ é uma ação aleatória entre as disponíveis para o estado atual.

\subsection{Problema do Caixeiro Viajante}

\subsubsection{Explicação do Problema}

O Problema do Caixeiro Viajante (PCV) é constituído de um conjunto de $N$ cidades que o agente deve visitar respeitando a restrição de passar somente uma vez por cada cidade. Com exceção da cidade final, a qual deve ser a mesma da inicial (Bodin, 1983, Pedro, 2013, Santos, 2014, Silva, 2014, Vitor, 2015, Ottoni et al., 2015, Ottoni, Nepomuceno and de Oliveira, 2016, Ottoni, Nepomuceno and Oliveira, 2016). Além disso, este problema pode receber a classificação de simétrico ou assimétrico. Para o caso simétrico, o custo final da trajetória realizada pelo agente não depende do sentido adotado. Em contrapartida, no caso assimétrico, o custo final está associado ao sentido adotado para à realização do percurso (Pedro, 2013, Santos, 2014, Ottoni, Nepomuceno and de Oliveira, 2016, Ottoni, Nepomuceno and Oliveira, 2016).

Algumas das aplicações deste problema são apresentadas por Goldbarg and Luna (2005) e Vitor (2015), dentre elas têm-se:

- Problemas de roteamento de veículos.

- Otimização do movimento de ferramentas de corte.

- Perfuração de placas de circuito impresso.

- Solução de problemas de sequenciamento de tarefas.

- Cortes em chapas de aço e vidro.

\subsubsection{Formulação}

A formulação descrita abaixo foi apresentada em Bodin (1983). No entanto, existem diversas formulações para o PCV e algumas delas podem ser encontradas em Goldbarg and Luna (2005) e Bodin (1983).

$$
\operatorname{Minimizar} \sum_{i=1}^{N} \sum_{j=1}^{N} c_{i j} x_{i j}
$$

sujeito a:

$$
\begin{gathered}
\sum_{i=1}^{N} x_{i j}=1 \quad(\forall j=1, \ldots, N) \\
\sum_{j=1}^{N} x_{i j}=1 \quad(\forall i=1, \ldots, N) \\
x_{i j} \in\{0,1\} \quad(\forall i, j=1, \ldots, N)
\end{gathered}
$$

$$
X=x_{i j} \in S \quad(\forall i, j=1, \ldots, N)
$$

A Eq. (4) representa a função objetivo do PCV. Nesta, $c_{i j}$ representa a distância entre os vértices $i$ e $j$. $x_{i j}$ indica se a aresta entre os vértices $i$ e $j$ faz parte da solução do problema. Em caso positivo, $x_{i j}$ assume o valor 1 . No entanto, caso a aresta não faça parte da solução, $x_{i j}$ assume $o$ valor 0. Além disto, as equações Eq. (5) e Eq. (6) garantem que cada vértice seja visitado pelo agente somente uma vez. A Eq. (7) garante que a variável $x_{i j}$ só possa assumir valores binários. Por fim, a Eq. (8) garante que não serão formadas sub-rotas para solução do problema (Bodin, 1983, Vitor, 2015, Ottoni, 2016, Ottoni, Ottoni, Oliveira and Nepomuceno, 2020).

\subsection{AutoML}

Dois dos principais pontos a serem definidos durante o Aprendizado de Máquina é qual algoritmo será utilizado e quais parâmetros este algoritmo irá receber (Tuggener et al., 2019). Normalmente, estas configurações iniciais são realizadas pelo usuário, porém para cada situação pode haver uma configuração específica que irá gerar um resultado melhor (Feurer et al., 2015). Assim, diversas configurações são testadas com o intuito de encontrar as melhores possíveis. No entanto, a definição dessas configurações inciais pode não ser tão óbvia e o experimentador pode não encontrar as configurações que forneceriam os melhores resultados (Mantovani et al., 2016). Neste sentido, temse o AutoML (Automated Machine Learning), que possui como finalidade principal a definição automatizada dos parâmetros e/ou algoritmos. Alguns de seus propósitos são (Brazdil et al., 2008, Hutter et al., 2018):

- Diminuição do esforço empregado pelo usuário durante a Aprendizagem de Máquina.

- Auxiliar o usuário durante a escolha do algoritmo adequado para cada problema.

- Aperfeiçoamento de desempenho dos algoritmos de Aprendizado de Máquina, dado que os parâmetros são ajustados de acordo com o problema.

O AutoML possui duas abordagens, a da otimização (Chen et al., 2019, Stamoulis et al., 2020) e a da recomendação (Mantovani et al., 2019, Cai et al., 2020). Um sistema AutoML que faz uso da recomendação é capaz de auxiliar o usuário durante a escolha de qual algoritmo deve ser utilizado (Brazdil et al., 2008, Mantovani et al., 2019). A depender do sistema desenvolvido, ele pode ainda sugerir ao usuário quais parâmetros devem ser aplicados, dado que os parâmetros podem interferir no desempenho do algoritmo (Brazdil et al., 2008, Hutter et al., 2014, Feurer et al., 2015, Mantovani et al., 2015, 2016, 2019). Já no caso de um sistema AutoML que utiliza a otimização, este sistema irá ajustar os parâmetros a fim de obter os que irão proporcionar o melhor resultado possível (Brazdil et al., 2008, Mantovani et al., 2015, Hutter et al., 2018).

Por conta de alguns fatores aplicar o AutoML pode ser complexo na prática (Brazdil et al., 2008, Mantovani et al., 
2016, Hutter et al., 2018, Mantovani et al., 2019):

- A avaliação de grandes conjuntos de dados pode ser complexa.

- O desenvolvimento de um sistema AutoML pode tornarse complexo.

- As configurações podem levar muito tempo para serem avaliadas.

Um dos principais assuntos dentro do Aprendizado de Máquina automatizado é o meta-aprendizado (metalearning) (Brazdil et al., 2008, Feurer et al., 2015, Hutter et al., 2018, Tuggener et al., 2019, Mantovani et al., 2019). No metalearning o sistema utiliza sua experiência adquirida em tarefas já realizadas para realizar novas tarefas (Feurer et al., 2015). Isto faz com que o esforço necessário para realizar a tarefa atual seja inferior ao esforço empregado para realizar as tarefas anteriores (Brazdil et al., 2008, Hutter et al., 2018). Não menos importante, um ponto a se ter atenção é que quanto maior a similaridade entre as tarefas (já realizadas e a serem realizadas), maiores são as chances de obter bons resultados (Hutter et al., 2018, Tuggener et al., 2019).

\section{Metodologia}

Nesta seção será apresentada a metodologia utilizada durante o desenvolvimento deste trabalho. A primeira subseção apresenta a modelagem de Aprendizado por Reforço selecionada para o Problema do Caixeiro Viajante. Já na subseção seguinte, é abordado acerca da Metodologia de Superfície de Resposta, método utilizado para implementar o AutoML. Na sequência, são apresentadas as etapas realizadas durante o experimento. Por fim, a última subseção apresenta o algoritmo de AutoML proposto por este trabalho.

\subsection{Modelagem do AR aplicado ao PCV}

Para solucionar o Problema do Caixeiro Viajante (PCV) através do Aprendizado por Reforço é necessário definir quais serão os estados, as ações e os reforços. Assim, após a análise de alguns trabalhos da literatura estes pontos foram definidos da seguinte maneira (Gambardella and Dorigo, 1995, Bianchi et al., 2009, Santos, 2009, Júnior et al., 2010, Ottoni, Ottoni, Oliveira and Nepomuceno, 2020):

- Estados: são todos os nós (cidades) do problema selecionado.

- Ações: são os estados ainda não visitados no estado atual.

- Reforços: após executar uma ação o agente irá receber um reforço que seguirá a seguinte regra:

$$
r_{i j}=-d_{i j} \text {, }
$$

sendo que, $r_{i j}$ representa o reforço recebido pela transição do estado $i$ para o estado $j$ e $d_{i j}$ representa a distância entre os respectivos estados (Ottoni, 2016).

\subsection{Metodologia de Superfície de Resposta}

A Metodologia de Superfície de Resposta, do inglês Response Surface Methodology (RSM), é uma técnica matemática que permite à aproximação de funções. Através do RSM, é possível otimizar processos que possuem múltiplos fatores que influenciam no resultado final. Geralmente, utiliza-se modelos de primeira ou segunda ordem para aproximar estas funções (Myers et al., 2016), conforme Eq. (10) e Eq. (11):

$$
\begin{gathered}
y=\beta_{0}+\beta_{1} x_{1}+\beta_{2} x_{2}+\varepsilon \\
y=\beta_{0}+\beta_{1} x_{1}+\beta_{2} x_{2}+\beta_{3} x_{1}^{2}+\beta_{4} x_{2}^{2}+\beta_{5} x_{1} x_{2}+\varepsilon
\end{gathered}
$$

A Eq. (10) representa o modelo de primeira ordem. Já Eq. (11) representa o modelo de segunda ordem. Nestas equações, $\beta$ n são os coeficientes, $\chi_{1}$ e $\chi_{2}$ são as variáveis independentes, $y$ é a variável resposta e $\varepsilon$ é o erro associado ao modelo ajustado (Ottoni et al., 2019).

Como foi proposto em Ottoni et al. (2018), neste trabalho será adotado o modelo de segunda ordem. Dado que, este modelo foi adotado em outros trabalhos na literatura e apresentou bons resultados. Após o ajuste da Eq. (11), a Eq. (12) representa a equação que será utilizada:

$$
y=\beta_{0}+\beta_{1} \alpha+\beta_{2} \gamma+\beta_{3} \alpha^{2}+\beta_{4} \gamma^{2}+\beta_{5} \alpha \gamma+\varepsilon
$$

na qual,

- $x_{1}=\alpha$

- $x_{2}=\gamma$

- y é a variável resposta que irá conter a distância total do trajeto realizado pelo agente.

\subsection{Planejamento dos Experimentos}

Inicialmente, foi definida qual modelagem do Aprendizado por Reforço seria aplicada ao Problema do Caixeiro Viajante juntamente com a linguagem que seria utilizada para desenvolver o sistema e qual algoritmo seria utilizado no mesmo. Assim, foi selecionado o algoritmo de Q-learning e este foi desenvolvido utilizando a linguagem R. Em seguida, foi selecionado qual valor de política $\epsilon$-greedy $(\epsilon)$ seria utilizado e quais valores de Taxa de Aprendizado $(\alpha)$ e Fator de Desconto $(\gamma)$ seriam utilizados para realização das combinações entre os parâmetros que seriam aplicados durante a primeira etapa do experimento. Os parâmetros (Taxa de Aprendizado e Fator de Desconto) selecionados para esta etapa inicial, foram aplicados em Ottoni (2016) e Ottoni et al. (2018). A Tabela 1 reúne estes parâmetros. Além disso, a mesma apresenta ainda algumas informações adicionais sobre a primeira etapa.

Já à Tabela 2, contém os dados pré-definidos da etapa final. Visando ajustar as configurações iniciais desta última etapa, a quantidade de episódios, de valores de parâmetros e combinações realizadas foram alterados. Entretanto, as 
Tabela 1: Dados iniciais da primeira etapa do experimento.

\begin{tabular}{ccc}
\hline & Quantidade & Valores \\
\hline$\alpha$ & 8 & 0,$01 ; 0,15 ; 0,30 ; 0,45 ; 0,60 ; 0,75 ; 0,90 ; 0,99$ \\
$\gamma$ & 8 & 0,$01 ; 0,15 ; 0,30 ; 0,45 ; 0,60 ; 0,75 ; 0,90 ; 0,99$ \\
$\epsilon$ & 1 & 0,01 \\
Combinações & $8 \times 8 \times 1=64$ & - \\
Épocas por Combinação & 5 & - \\
Episódios por Época & 1000 & - \\
Episódios por Combinação & $5 \times 1000=5000$ & - \\
Total de Épocas & $5 \times 64=320$ & - \\
Total de Episódios & $1000 \times 320=320000$ & \\
\hline
\end{tabular}

demais configurações não necessitaram de ajuste, sendo assim, as mesmas foram mantidas.

Tabela 2: Dados iniciais da etapa final do experimento.

\begin{tabular}{c|c|c}
\hline & Quantidade & Valores \\
\hline$\alpha$ & 1 & - \\
$\epsilon$ & 1 & - \\
Combinações & 1 & 0,01 \\
Épocas por Combinação & $1 \times 1 \times 1=1$ & - \\
Episódios por Época & 10000 & - \\
Episódios por Combinação & $5 \times 10000=50000$ & - \\
Total de Épocas & 5 & - \\
Total de Episódios & $5 \times 10000=50000$ & - \\
\hline
\end{tabular}

As instâncias selecionadas para o experimento foram obtidas na biblioteca TSPLIB ${ }^{1}$ (Reinelt, 1991). Na TSPLIB podem ser encontrados diversos problemas de otimização, a biblioteca oferece dados do Problema do Caixeiro Viajante e de outros problemas relacionados a ele (Reinelt, 1995, Bianchi, 2004, Santos, 2014). Alguns dos problemas que podem ser encontrados na TSPLIB são seguintes seguimentos (Reinelt, 1995):

- Symmetric traveling salesman problem (TSP): problemas do caixeiro viajante simétrico.

- Asymmetric traveling salesman problem (ATSP): problemas do caixeiro viajante assimétrico.

Assim, foram selecionados problemas simétricos e assimétricos do PCV. Tais problemas estão reunidos na Tabela 3 .

\subsection{Algoritmo Proposto}

O sistema de AutoML proposto, denominado AutoRL-TSPRSM, foi implementado na linguagem R. Neste, foi realizada a implementação manual de grande parte dos processos realizados, como do algoritmo Q-learning por exemplo. Associado a isto, fez-se ainda o uso de bibliotecas e funções disponíveis na linguagem $\mathrm{R}$, como por exemplo a biblioteca ' $r s m$ ' e as funções anova, summary e ks.test. Não menos importante, vale ressaltar que o algoritmo desenvolvido faz uso de todas as informações já apresentadas anteriormente, nas etapas inicial e final é utilizada a mo-

\footnotetext{
${ }^{1}$ http://comopt.ifi.uni-heidelberg.de/software/TSPLIB95/
}

Tabela 3: Problemas selecionados da TSPLIB.

\begin{tabular}{clcr}
\hline Tipo & Problema & Cidades & Ótimo \\
\hline \multirow{4}{*}{ TSP } & swiss42 & 42 & 1273 \\
& eil51 & 51 & 426 \\
& berlin52 & 52 & 7542 \\
& st70 & 70 & 675 \\
& eil76 & 76 & 538 \\
& pr76 & 76 & 108159 \\
& rat99 & 99 & 1211 \\
& kroa100 & 100 & 21282 \\
& eil101 & 101 & 629 \\
& bier127 & 127 & 118282 \\
& ch130 & 130 & 6110 \\
\hline \multirow{4}{*}{ ATSP } & ftv33 & 34 & 1286 \\
& p43 & 43 & 5620 \\
& ftv44 & 45 & 1613 \\
& ftv47 & 48 & 1776 \\
& ry48p & 48 & 14422 \\
& ft53 & 53 & 6905 \\
& ftv64 & 65 & 1839 \\
& ft70 & 70 & 38673 \\
\hline
\end{tabular}

delagem do Aprendizado por Reforço selecionada para o problema. Já para à automatização, é aplicada a Metodologia de Superfície de Resposta. O sistema desenvolvido pode ser utilizado tanto com instâncias simétricas quanto com instâncias assimétricas. O método AutoRL-TSP-RSM utiliza como métrica durante a modelagem o menor valor de distância de rota por época alcançado dentre os resultados da primeira etapa.

O Algoritmo 1 descreve o algoritmo Q-learning que foi implementado, este algoritmo é utilizado durante a primeira e a última etapa. Na primeira etapa, os valores de fator de desconto e taxa de aprendizado recebidos pela função são apresentados na Tabela 1. Já para à última etapa, os parâmetros serão definidos pelo sistema utilizando a função descrita no Algoritmo 2.

O método utilizado para à automatização do sistema é a Metodologia de Superfície de Resposta. Inicialmente, é gerado o modelo de regressão linear múltipla, em seguida alguns dados são extraídos do modelo ajustado para à realização de algumas validações. Em uma destas verificações avalia-se os resíduos do modelo gerado, assim, esta validação tem como objetivo verificar se os resíduos do modelo seguem uma distribuição normal. Além disto, é realizada também a verificação do nível de significância do modelo ajustado. Não menos importante, avalia-se ainda se os valores obtidos para $\alpha$ e $\gamma$ utilizando RSM respeitam os 

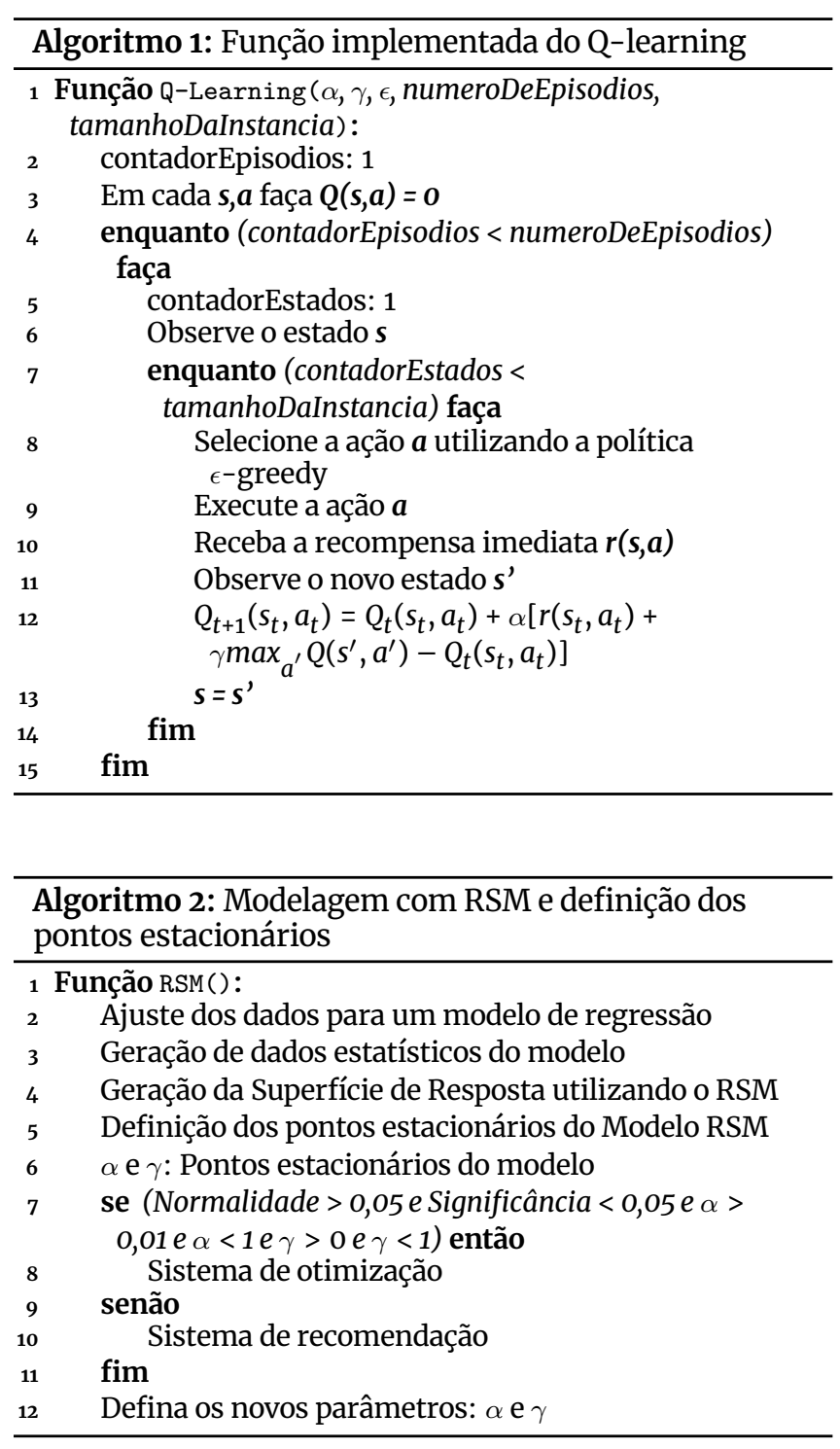

limites definidos ( $\alpha$ e $\gamma$ devem variar somente entre 0 e 1 ). Caso todas verificações sejam satisfeitas, o sistema seguirá a vertente da otimização e utilizará na etapa final os pontos estacionários da superfície gerada como parâmetros. Do contrário, o sistema seguirá a vertente da recomendação e utilizará na etapa final os parâmetros que proporcionaram o melhor desempenho durante a etapa inicial. O Algoritmo 2 apresenta estas etapas.

Finalmente, o Algoritmo 3 faz uso dos Algoritmos 1 e 2, sendo assim, o mesmo representa o sistema implementado. Primeiramente, são definidos os dados que serão fixos durante a execução do sistema. A primeira etapa utiliza o Algoritmo 1 e os dados obtidos nesta etapa são armazenados para serem utilizados posteriormente pelo sistema nas etapas seguintes. Já na segunda etapa, é utilizado o Algoritmo 2 e através dele são definidos os novos valores de taxa de aprendizado e fator de desconto que serão aplicados na etapa final. Por último, o sistema utiliza novamente o algoritmo 1, no entanto, nesta etapa os valores de fator de desconto e taxa de aprendizado serão os

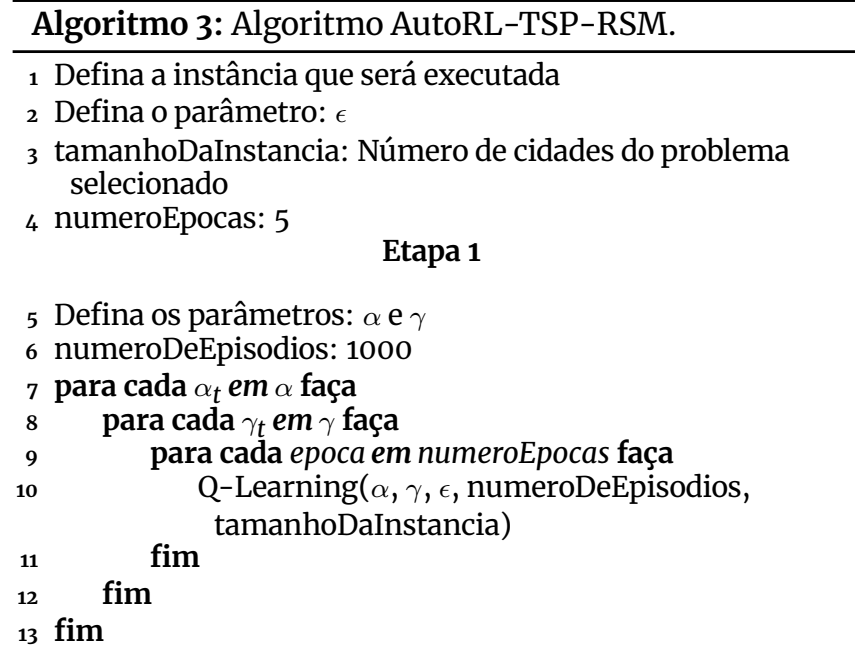

Etapa 2

$14 \operatorname{RSM}()$

Etapa 3

15 numeroDeEpisodios: 10000

16 para cada epoca em numeroEpocas faça

17 Q-Learning $(\alpha, \gamma, \epsilon$, numeroDeEpisodios,

18 fim tamanhoDaInstancia)

definidos automaticamente na etapa anterior pelo próprio sistema.

\subsection{Análise dos Resultados}

A seção seguinte apresentará os resultados obtidos com este trabalho. Para tal, segue-se as seguintes etapas:

i. Análise das Medidas de Adequação obtidas. Neste trabalho irá analisar-se os coeficientes de determinação múltipla, o nível de significância e o índice de normalidade.

ii. Verificação dos Coeficientes Ajustados. Será verificado o nível de significância obtido para cada coeficiente ajustado para à Eq. (12).

iii. Análise dos Parâmetros Ajustados. Nesta seção serão apresentados os parâmetros ajustados pelo sistema proposto. Além disto, é avaliado se existe alguma relação que parametrize a definição dos parâmetros.

iv. Comparação com parâmetros da literatura. Nesta seção serão apresentados os resultados obtidos ao aplicar os parâmetros ajustados pelo sistema e ao aplicar alguns parâmetros da literatura. Além disso, será realizada uma comparação entre estes resultados.

\section{Resultados}

Esta seção apresenta os resultados obtidos neste trabalho. Inicialmente, são apresentadas as medidas de adequação obtidas durante o experimento. Na sequência, são apre- 
sentados os coeficientes ajustados durante o experimento. Em seguida, apresenta-se os parâmetros ajustados pelo sistema proposto. Por fim, é apresentada uma comparação realizada entre resultados obtidos ao aplicar os parâmetros ajustados pelo sistema proposto e os parâmetros obtidos da literatura.

\subsection{Medidas de Adequação}

Como forma de análise dos modelos gerados pelo sistema, algumas propriedades de cada instância testada foram avaliadas. Assim, foram extraídos de cada modelo a normalidade, a significância, o coeficiente de determinação múltipla $\left(R_{a}\right)$ e o coeficiente de determinação múltipla ajustado $\left(R_{a}^{2}\right)$. Todos estes dados foram extraídos utilizando a função sumamry do R.

Os coeficientes $R_{a}$ e $R_{a}^{2}$, expressam o quão bom é o modelo ajustado e o valor de ambas as métricas deve variar entre o e 1. Assim, quanto maior for o valor destas métricas, melhor é o modelo. Contudo, o valor de $R_{a}$ pode aumentar por conta da adição de novos termos no modelo. Desta forma, faz-se necessário também a avaliação de $R_{a}^{2}$ para verificar a qualidade do modelo ajustado (Myers et al., 2016).

Para avaliar a normalidade dos resíduos dos modelos, foi selecionado o teste de Kolmogorov-Smirnov (KS). Uma vez que o mesmo é dos testes mais utilizados para verificar se os dados seguem uma distribuição normal (Leotti et al., 2005, Razali et al., 2011). Avaliar a normalidade dos dados é de extrema importância, sendo que, quando não é possível comprovar a normalidade dos dados, pode ser que estes dados não sejam confiáveis. $\mathrm{O}$ teste KS avalia hipóteses e define se a hipótese é verdadeira ou não, assim, têm-se a hipótese inicial $\left(H_{0}\right)$ e a hipótese alternativa $\left(H_{a}\right)$ (Razali et al., 2011):

$\left\{H_{0}:\right.$ Os dados seguem uma distribuição normal.

$H_{a}$ : Os dados não seguem uma distribuição normal.

O índice de normalidade dos resíduos dos modelos foi definido como maior que $5 \%$. Neste caso, aceita-se $H_{0}$ e os dados possuem distribuição normal. Do contrário, aceita-se $H_{a}\left(p-\right.$ valor $\left._{K S}<0,05\right)$ e os dados não possuem distribuição normal.

Assim como a avaliação da normalidade, a avaliação da significância também é realizada por meio de hipóteses. Igualmente, têm-se a hipótese inicial $\left(H_{0}\right)$ e a hipótese alternativa $\left(H_{a}\right)$. $O$ índice de significância indica se as variáveis possuem relação com a variável resposta do modelo ajustado (Myers et al., 2016).

$\left\{H_{0}:\right.$ O não modelo é significativo.

$H_{a}$ : O modelo é significativo.

O nível de significância foi definido como $5 \%$, neste caso o modelo é significativo e aceita-se $H_{a}$. Já em caso contrário, o modelo é dito não significativo e aceita-se $H_{0}$ ( $p$-valor > 0,05).

A Tabela 4 apresenta as medidas de adequação obtidas para cada instância utilizada. Assim, na mesma pode-se verificar que as instâncias swiss42, eil51, berlin52, st70, eil76, rat99, kroa100, eil101, bier127, ftv33, ftv44, ftv47, ry48p, ft53, ftv64 e ftv70 atenderam as condições que foram avaliadas durante o experimento. Dessa forma, du-
Tabela 4: Medidas de adequação obtidas durante o experimento.

\begin{tabular}{llcccc}
\hline Tipo & Problema & $p-$ valor $_{K S}$ & p-valor & $\boldsymbol{R}^{\mathbf{2}}$ & $\boldsymbol{R}_{\boldsymbol{a}}^{\mathbf{2}}$ \\
\hline \multirow{6}{*}{ TSP } & swiss42 & 0,149 & 0,000 & 0,728 & 0,723 \\
& eil51 & 0,430 & 0,000 & 0,676 & 0,671 \\
& berlin52 & 0,063 & 0,000 & 0,708 & 0,703 \\
& st70 & 0,065 & 0,000 & 0,709 & 0,704 \\
& eil76 & 0,404 & 0,000 & 0,726 & 0,722 \\
& pr76 & 0,000 & 0,000 & 0,621 & 0,615 \\
& rat99 & 0,707 & 0,000 & 0,689 & 0,684 \\
& kroa100 & 0,058 & 0,000 & 0,762 & 0,758 \\
& eil101 & 0,683 & 0,000 & 0,761 & 0,758 \\
& bier127 & 0,526 & 0,000 & 0,792 & 0,788 \\
& ch130 & 0,035 & 0,000 & 0,796 & 0,793 \\
\hline \multirow{4}{*}{ ATSP } & ftv33 & 0,322 & 0,000 & 0,704 & 0,699 \\
& p43 & 0,026 & 0,000 & 0,496 & 0,488 \\
& ftv44 & 0,369 & 0,000 & 0,651 & 0,645 \\
& ftv47 & 0,547 & 0,000 & 0,686 & 0,681 \\
& ry48p & 0,065 & 0,000 & 0,690 & 0,685 \\
& ft53 & 0,534 & 0,000 & 0,707 & 0,400 \\
& ftv64 & 0,794 & 0,000 & 0,700 & 0,695 \\
& ft70 & 0,290 & 0,000 & 0,788 & 0,785 \\
\hline
\end{tabular}

rante a etapa final foram aplicados os pontos estacionários da superfície de resposta como parâmetros. Em contrapartida, as instâncias pr76, ch130 e p43 não atenderam aos critérios definidos, consequentemente, o sistema aplicou na etapa final os parâmetros que proporcionaram os melhores resultados de distância de rota durante a primeira etapa.

\subsection{Coeficientes Ajustados}

A Tabela 5 reúne os coeficientes ajustados durante o experimento para à Eq. (12) de todos os problemas utilizados durante o experimento. Nesta, os coeficientes são representados por $\beta_{0}, \beta_{1}, \beta_{2}, \beta_{3}, \beta_{4}$ e $\beta_{5}$. Não menos importante, ressalta-se que o nível de significância desejado é o mesmo definido na seção anterior.

A Tabela 6 por sua vez, apresenta as significâncias obtidas para cada coeficiente ajustado. Sendo que, $p_{0}$ indica a significância de $\beta_{0}, p_{1}$ a significância de $\beta_{1}$ e assim continuamente até que finalmente $p_{5}$ indica a significância de $\beta_{5}$. Assim como na seção anterior, os dados desta seção também foram obtidos utilizando a função sumamry do R.

À aplicação da Metodologia de Superfície de Resposta permite realizar análises através de gráficos dos dados de estudo. Estas análises podem ser realizadas em gráficos de contorno ou em superfícies de resposta. Sendo que, no caso dos gráficos de contorno utiliza-se o espaço bidimensional e nas superfícies de respostas utiliza-se o espaço tridimensional (Myers et al., 2016).

Diante do dados apresentados na Tabela 6, percebese que os coeficientes $\beta_{0}, \beta_{1}$ e $\beta_{3}$ cumpriram o nível de significância desejado, logo, pode-se constatar que estes coeficientes são significantes para os modelos ajustados. Já os demais coeficientes, obtiveram $p$-valor $>0,05 \mathrm{em}$ alguns dos modelos ajustados, no entanto, os mesmos foram mantidos com a finalidade de preservar um modelo padrão de sistema AutoML para o experimento. 
Tabela 5: Coeficientes ajustados durante o experimento.

\begin{tabular}{lrrrrrr}
\hline Problema & $\boldsymbol{\beta}_{\mathbf{0}}$ & $\boldsymbol{\beta}_{\mathbf{1}}$ & $\boldsymbol{\beta}_{\mathbf{2}}$ & $\boldsymbol{\beta}_{\mathbf{3}}$ & $\boldsymbol{\beta}_{\mathbf{4}}$ & $\boldsymbol{\beta}_{\mathbf{5}}$ \\
\hline Swiss42 & 2071,92 & $-2170,38$ & $-249,51$ & 1646,53 & 547,45 & $-127,11$ \\
eil51 & 710,96 & $-692,58$ & $-152,25$ & 520,01 & 295,15 & $-80,49$ \\
berlin52 & 12326,50 & $-13782,60$ & $-495,60$ & 10077,20 & 1397,70 & 712,60 \\
st70 & 1212,51 & $-1675,09$ & $-166,73$ & 1290,83 & 422,63 & $-143,95$ \\
eil76 & 1004,72 & $-1390,44$ & $-333,26$ & 1059,62 & 577,51 & $-102,06$ \\
pr76 & 153855,00 & $-66994,00$ & $-32407,00$ & 45932,00 & 44100,00 & 6611,00 \\
rat99 & 2098,29 & $-2111,59$ & $-1047,57$ & 1489,62 & 1410,95 & 186,59 \\
kroa100 & 47983,00 & $-78735,00$ & $-6775,00$ & 60500,00 & 18084,00 & $-8185,00$ \\
eil101 & 1333,94 & $-1883,11$ & $-332,40$ & 1401,07 & 617,40 & $-156,20$ \\
bier127 & 251936,00 & $-397990,00$ & $-30905,00$ & 293326,00 & 74918,00 & $-4089,00$ \\
ch130 & 14443,70 & $-26097,20$ & $-1101,90$ & 19883,00 & 3610,70 & $-1468,40$ \\
ftv33 & 2045,37 & $-1998,34$ & 33,23 & 1532,51 & 248,00 & $-79,03$ \\
p43 & 5676,19 & $-63,41$ & 5,72 & 36,39 & $-2,46$ & 15,31 \\
ftv44 & 2976,40 & $-3376,92$ & $-1007,73$ & 2488,68 & 1511,80 & $-28,98$ \\
ftv47 & 3248,39 & $-3472,71$ & $-911,58$ & 2570,78 & 1575,64 & $-93,70$ \\
ry48p & 22921,40 & $-23333,70$ & $-4922,00$ & 17365,10 & 9276,30 & $-1312,60$ \\
ft53 & 10792,90 & $-6736,60$ & $-3538,20$ & 4976,30 & 5090,10 & $-762,50$ \\
ftv64 & 3948,60 & $-5436,80$ & $-1437,30$ & 4098,20 & 2378,80 & $-359,10$ \\
ft70 & 47105,04 & $-13744,19$ & $-3969,24$ & 8616,60 & 9086,44 & $-91,43$ \\
\hline
\end{tabular}

Tabela 6: Significância dos coeficientes ajustados.

\begin{tabular}{lcccccc}
\hline Problema & $p_{\mathbf{0}}$ & $p_{\mathbf{1}}$ & $p_{\mathbf{2}}$ & $p_{\mathbf{3}}$ & $p_{4}$ & $p_{5}$ \\
\hline swiss42 & 0,0000 & 0,0000 & 0,0259 & 0,0000 & 0,0000 & 0,1175 \\
eil51 & 0,0000 & 0,0000 & 0,0011 & 0,0000 & 0,0000 & 0,0172 \\
berlin52 & 0,0000 & 0,0000 & 0,4580 & 0,0000 & 0,0180 & 0,1420 \\
st70 & 0,0000 & 0,0000 & 0,0688 & 0,0000 & 0,0000 & 0,0308 \\
eil76 & 0,0000 & 0,0000 & 0,0000 & 0,0000 & 0,0000 & 0,0688 \\
pr76 & 0,0000 & 0,0000 & 0,0000 & 0,0000 & 0,0000 & 0,0884 \\
rat99 & 0,0000 & 0,0000 & 0,0000 & 0,0000 & 0,0000 & 0,0700 \\
kroa100 & 0,0000 & 0,0000 & 0,0731 & 0,0000 & 0,0000 & 0,0030 \\
eil101 & 0,0000 & 0,0000 & 0,0005 & 0,0000 & 0,0000 & 0,0235 \\
bier127 & 0,0000 & 0,0000 & 0,0648 & 0,0000 & 0,0000 & 0,7360 \\
ch130 & 0,0000 & 0,0000 & 0,2955 & 0,0000 & 0,0001 & 0,0555 \\
ftv33 & 0,0000 & 0,0000 & 0,7516 & 0,0000 & 0,0077 & 0,3005 \\
p43 & 0,0000 & 0,0000 & 0,3222 & 0,0000 & 0,6284 & 0,0003 \\
ftv44 & 0,0000 & 0,0000 & 0,0000 & 0,0000 & 0,0000 & 0,8570 \\
ftv47 & 0,0000 & 0,0000 & 0,0000 & 0,0000 & 0,0000 & 0,5580 \\
ry48p & 0,0000 & 0,0000 & 0,0007 & 0,0000 & 0,0000 & 0,2119 \\
ft53 & 0,0000 & 0,0000 & 0,0000 & 0,0000 & 0,0000 & 0,0544 \\
ftv64 & 0,0000 & 0,0000 & 0,0000 & 0,0000 & 0,0000 & 0,1290 \\
ft70 & 0,0000 & 0,0000 & 0,0000 & 0,0000 & 0,0000 & 0,8970 \\
\hline
\end{tabular}

\subsubsection{Superfície de Resposta e Gráfico de contorno}

A Fig. 1 apresenta o gráfico de contorno do problema berlin52. Já a Fig. 2, mostra a superfície de resposta deste mesmo problema. Sendo que, ambos os gráficos foram obtidos durante o experimento com o algoritmo de AutoML proposto.

Na Fig. 1 e na Fig. 2, pode-se verificar para quais faixas de valores de taxa de aprendizado e fator de desconto a distância final da rota tende a ser minimizada. Sendo que, em ambos os gráficos estas regiões são representadas com tons mais avermelhados, neste caso, a distância final da rota tende a ser minimizada quando são aplicados aproximadamente os valores de $0,6 \leq \alpha \leq 0,8$ e $0 \leq \gamma \leq 0,3$.

\subsection{Parâmetros Ajustados}

Através da Metodologia de Superfície de Resposta é possível modelar diversos tipos de problemas e pode-se ainda obter os pontos estacionários de cada modelo gerado. Os pontos estacionários, são responsáveis por representar o local de mínimo, máximo ou ponto de sela do modelo (Riboldi and Nascimento, 1994). A Tabela 7, reúne os parâmetros ajustados para cada modelo gerado por meio da Metodologia de Superfície de Resposta. Para os casos em que o modelo atendeu medidas de adequação são apresentados os pontos estacionários das superfícies obtidas. Já nos casos em que o modelo gerado não atendeu à alguma das verificações, são apresentados os valores de Taxa de Aprendizado e Fator de desconto quê quando combinados proporcionaram o melhor resultado de distância total de rota na primeira etapa do experimento. Vale ressaltar quê estes valores foram determinados através do algoritmo de AutoRL proposto durante a segunda etapa do experimento.

Ao verificar a Tabela 7, pode-se perceber que os valores de Taxa de Aprendizado e Fator de desconto obtidos para cada problema analisado foi diferente. Sendo assim, pode-se perceber que não há um padrão para definição dos 


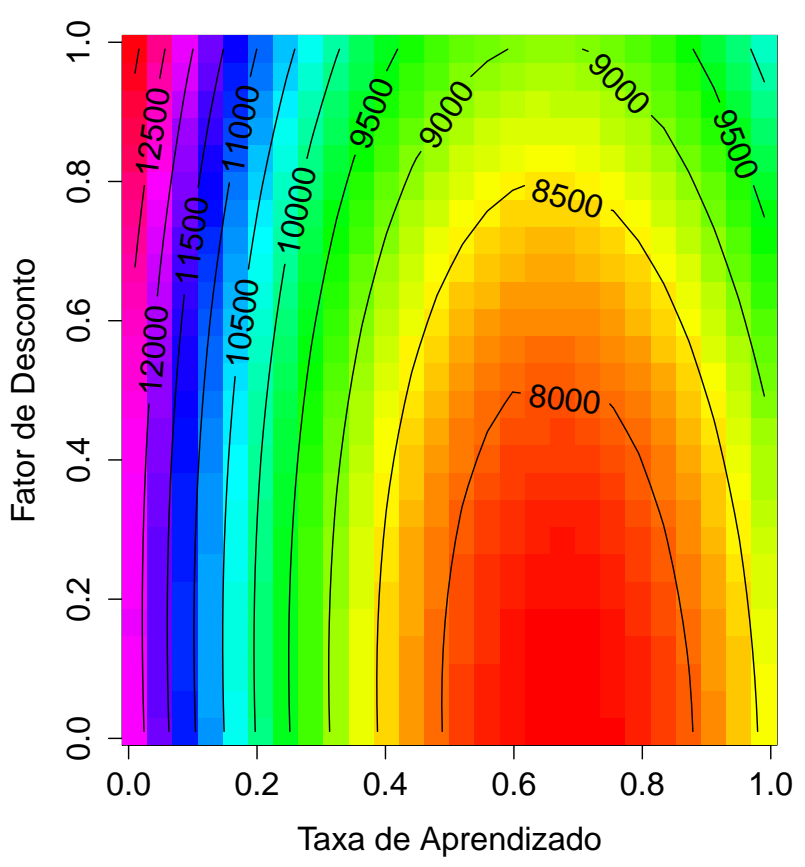

Figura 1: Gráfico de contorno do problema berlin52 gerado pelo algoritmo de AutoML proposto utilizando os parâmetros $\alpha=0,684 \mathrm{e} \gamma=0,003$.

Tabela 7: Parâmetros ajustados pelo algoritmo de AutoML proposto.

\begin{tabular}{|c|c|c|}
\hline Problema & $\alpha$ & $\gamma$ \\
\hline swiss42 & 0,671 & 0,306 \\
\hline eil51 & 0,693 & 0,352 \\
\hline berlin52 & 0,684 & 0,003 \\
\hline st70 & 0,666 & 0,311 \\
\hline eil76 & 0,673 & 0,348 \\
\hline pr76 & 0,990 & 0,900 \\
\hline rat99 & 0,688 & 0,326 \\
\hline kroa10o & 0,674 & 0,340 \\
\hline eil101 & 0,692 & 0,357 \\
\hline bier127 & 0,681 & 0,225 \\
\hline ch130 & 0,990 & 0,150 \\
\hline $\mathrm{ftv} 33$ & 0,653 & 0,037 \\
\hline $0 / 3$ & 0,990 & 0,010 \\
\hline $\mathrm{ftv} 44$ & 0,680 & 0,341 \\
\hline ftv47 & 0,681 & 0,309 \\
\hline ry $48 p$ & 0,684 & 0,314 \\
\hline $\mathrm{ft} 53$ & 0,707 & 0,400 \\
\hline ftv64 & 0,679 & 0,353 \\
\hline $\mathrm{ft70}$ & 0,799 & 0,222 \\
\hline
\end{tabular}

parâmetros sendo necessária a realização de uma avaliação para realizar o ajuste de parâmetros necessário para cada problema avaliado.

A Fig. 3 exibe a rota final obtida para a instância st70 aplicando os parâmetros definidos de maneira automática na segunda etapa do experimento, neste caso, os parâmetros utilizados foram os pontos estacionários do modelo

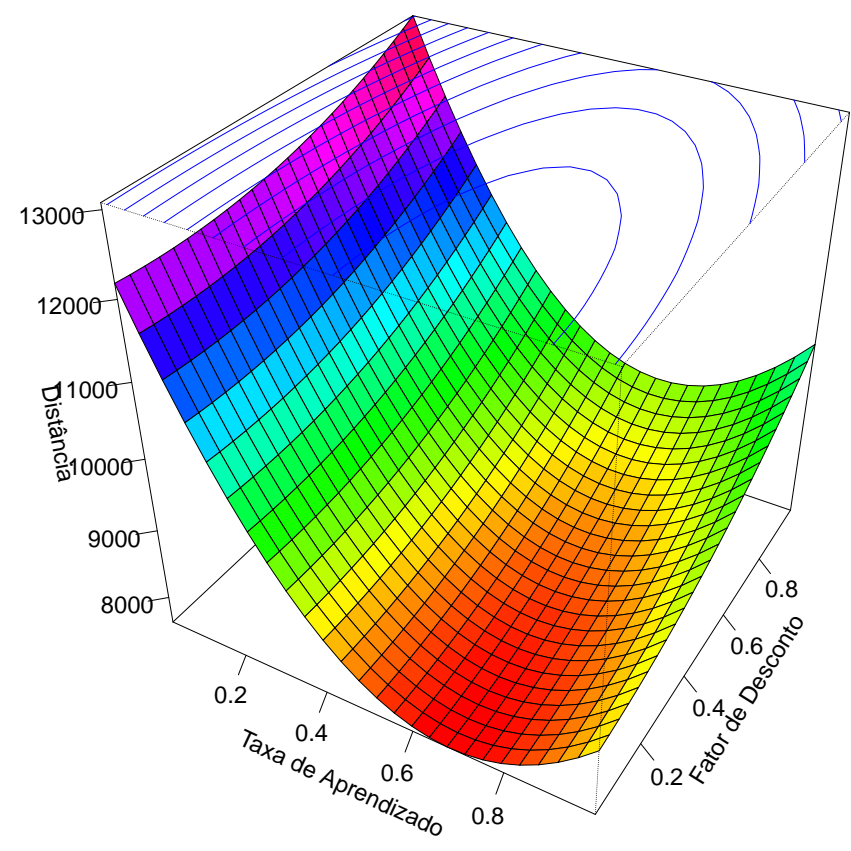

Figura 2: Superfície de resposta do problema berlin52 gerada pelo algoritmo de AutoML proposto utilizando os parâmetros $\alpha=0,684 \mathrm{e} \gamma=0,003$.

ajustado para à superfície de resposta gerada. Vale ressaltar quê este gráfico foi obtido automaticamente através do algoritmo proposto durante a última etapa do experimento.

\subsection{Comparação com parâmetros de outros traba- lhos}

A Tabela 8 apresenta os resultados obtidos em uma nova rodada de experimentos. Para legitimar os resultados já apresentados e a importância do assunto que é estudado neste artigo, decidiu-se pela realização de uma nova rodada de experimentos. Para tal, foram selecionados alguns trabalhos que aplicaram o Aprendizado por Reforço no Problema do Caixeiro Viajante. Nesse sentido, foram selecionados os trabalhos de Gambardella and Dorigo (1995), Sun et al. (2001), Liu and Zeng (2009) e Santos (2009), e destes trabalhos foram extraídos os seguintes parâmetros:

- $\alpha=0,1 \mathrm{e} \gamma=0,3$ (Gambardella and Dorigo, 1995).

- $\alpha=0,1 \mathrm{e} \gamma=0,9$ (Liu and Zeng, 2009).

- $\alpha=0,8$ e $\gamma=0,9$ (Sun et al., 2001).

- $\alpha=0,8$ e $\gamma=1$ (Santos, 2009).

Para à realização desta novo ciclo, foram executadas 5 épocas sendo que cada época contou com 10.000 episódios. Cada um dos parâmetros selecionados foram testados nessas condições. Além disso, neste novo ciclo foram utilizados também os parâmetros definidos pelo sistema proposto, sendo que estes parâmetros são apresentados 
Tabela 8: Resultados da aplicação dos parâmetros definidos pelo sistema de AutoML e dos parâmetros selecionados de outros trabalhos, sendo AutoML: resultados obtidos com os parâmetros definidos através do sistema de AutoML; So9: resultados com parâmetros de Santos (2009); G95: resultados com parâmetros de Gambardella and Dorigo (1995); L09: resultados com parâmetros de Liu and Zeng (2009); So1: resultados com parâmetros de Sun et al. (2001).

\begin{tabular}{lrrrrrr}
\hline Problema & Ótimo & AutoML & So9 & G95 & L09 & S01 \\
\hline swiss42 & 1273 & $\mathbf{1 3 3 5}$ & 1689 & 1364 & 1564 & 1435 \\
eil51 & 426 & $\mathbf{4 7 5}$ & 614 & 478 & 505 & 497 \\
berlin52 & 7542 & $\mathbf{7 8 7 1}$ & 9620 & 8422 & 8731 & 8665 \\
st70 & 675 & $\mathbf{7 0 4}$ & 1032 & 712 & 765 & 780 \\
eil76 & 538 & 567 & 791 & 570 & 587 & 595 \\
pr76 & 108159 & 120665 & 150757 & 123123 & 119189 & $\mathbf{1 1 7 9 1 4}$ \\
rat99 & 1211 & $\mathbf{1 3 5 0}$ & 2093 & 1373 & 1372 & 1419 \\
kroa100 & 21282 & 24555 & 35347 & $\mathbf{2 4 2 7 8}$ & 27552 & 26626 \\
eil101 & 629 & $\mathbf{7 0 7}$ & 981 & 732 & 728 & 716 \\
bier127 & 118282 & $\mathbf{1 2 6 9 2 2}$ & 169424 & 129382 & 144413 & 146748 \\
ch130 & 6110 & $\mathbf{6 5 9 0}$ & 7659 & 6854 & 7702 & 7516 \\
ftv33 & 1286 & $\mathbf{1 3 6 5}$ & 1795 & 1472 & 1556 & 1578 \\
p43 & 5620 & $\mathbf{5 6 3 7}$ & 5647 & 5652 & 5651 & 5648 \\
ftv44 & 1613 & 1852 & 2755 & 1876 & $\mathbf{1 8 5 0}$ & 1867 \\
ftv47 & 1776 & $\mathbf{2 0 8 7}$ & 2993 & 2100 & 2266 & 2157 \\
ry48p & 14422 & $\mathbf{1 5 5 7 5}$ & 19899 & $\mathbf{1 5 5 7 5}$ & 16775 & 16480 \\
ft53 & 6905 & 8182 & 9263 & 8287 & 7945 & $\mathbf{7 8 9 5}$ \\
ftv64 & 1839 & $\mathbf{2 1 0 0}$ & 3433 & 2130 & 2333 & 2286 \\
ft70 & 38673 & 41568 & 47847 & $\mathbf{4 1 3 8 2}$ & 42533 & 42040 \\
\hline
\end{tabular}

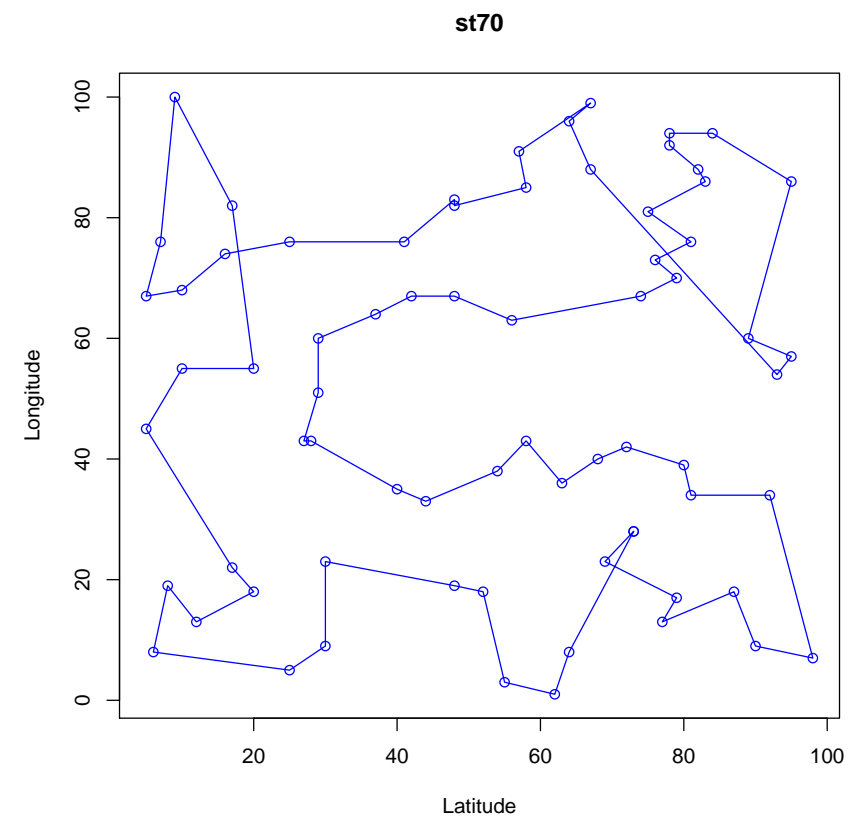

Figura 3: Rota final obtida para o problema st70 com o algoritmo de AutoML proposto utilizando os parâmetros $\alpha=0,666$ e $\gamma=0,311$. Distância final: 707.

na Tabela 7. Visando assim uma comparação imparcial e justa dentre todos os parâmetros.

Como forma de organização, alguns ajustes foram realizados nos títulos das colunas da Tabela 8. Nessa linha, foi utilizada a seguinte regra para definição dos títulos das colunas que apresentam os resultados obtidos com os parâmetros de outros trabalhos: primeira letra do autor seguido dos dois últimos dígitos correspondentes ao ano do trabalho. Assim, Santos (2009) será representado por 'S09', Gambardella and Dorigo (1995) por 'G95', Liu and Zeng (2009) por 'L09' e Sun et al. (2001) por 'S01'. No que se refere a coluna que apresenta os resultados obtidos com os parâmetros definidos com o sistema de AutoML proposto por este trabalho, esta foi nomeada com o título de 'AutoML'.

A partir dos resultados apresentados na Tabela 8, podese perceber que as instâncias swiss42, eil51, berlin52, st70, eil76, rat99, eil101, berlin127, ch130, ftv33, p43, ftv47, ftv64 obtiveram um melhor resultado quando foram aplicados os parâmetros definidos pelo sistema de AutoML. Já quando foram aplicados os parâmetros de Gambardella and Dorigo (1995), as instâncias kroa100 e ft70, apresentaram um melhor desempenho. Quando foram utilizados os parâmetros de Sun et al. (2001), obteve-se o melhor resultado em pr76 e ft53. Ao utilizar os parâmetros de Liu and Zeng (2009), a instância ftv44 apresentou o melhor desempenho. Por fim, tratando-se dos parâmetros extraídos de Santos (2009), nenhuma instância apresentou o melhor resultado. Não menos importante, vale ressaltar ainda quê a instância ry $48 \mathrm{p}$ convergiu igualmente para o melhor resultado tanto quando foram aplicados os parâmetros definidos pelo sistema quanto quando foram aplicados os parâmetros selecionados do trabalho de Gambardella and Dorigo (1995).

\section{Conclusão}

O objetivo deste trabalho foi propor um sistema de Aprendizado por Reforço Automatizado (AutoRL-TSP-RSM) com Metodologia de Superfície de Resposta para o Problema do Caixeiro Viajante. O sistema proposto tem como intuito ajustar de forma automática os parâmetros de taxa de aprendizado e fator de desconto do algoritmo Q- 
learning. Para isso, foram utilizados conceitos e realizados avanços em relação a trabalhos recentes (Ottoni et al., 2018, Ottoni, Ottoni, Oliveira and Nepomuceno, 2020).

A partir do sistema de AutoRL-TSP-RSM proposto foi possível ajustar parâmetros para 20 instâncias da TSPLIB de forma automática. Os resultados revelaram que, em geral, as menores de distâncias de rota foram alcançadas ao aplicar os valores definidos pelo sistema de AutoML, em comparação com parâmetros definidos na literatura.

Em trabalhos futuros, espera-se experimentar o sistema desenvolvido com outros problemas da TSPLIB e outros problemas de otimização combinatória. Além disso, espera-se ainda evoluir o sistema para indicação de algoritmos e para o ajuste do parâmetro Política $\epsilon$-greedy.

\section{Referências Bibliográficas}

Alipour, M., Razavi, S., Feizi Derakhshi, M. and Balafar, M. (2018). A hybrid algorithm using a genetic algorithm and multiagent reinforcement learning heuristic to solve the traveling salesman problem, Neural Computing and Applications 30(9): 2935-2951. http: //dx.doi.org/10.1007/s00521-017-2880-4.

Alzubaidi, L., Zhang, J., Humaidi, A., Al-Dujaili, A., Duan, Y., Al-Shamma, O., Santamaría, J., Fadhel, M., AlAmidie, M. and Farhan, L. (2021). Review of deep learning: concepts, cnn architectures, challenges, applications, future directions, Journal of Big Data 8(1). http://dx.doi.org/10.1186/s40537-021-00444-8.

Bianchi, R. A. C., Ribeiro, C. H. C. and Costa, A. H. R. (2009). On the relation between ant colony optimization and heuristically accelerated reinforcement learning, 1st international workshop on hybrid control of autonomous system, Citeseer, pp. 49-55. Disponível em http://citeseerx.ist.psu.edu/viewdoc/download? do $i=10.1 \cdot 1 \cdot 605.9537 \& r e p=r e p 1 \&$ type=pdf.

Bianchi, R. A. d. C. (2004). Uso de heurísticas para a aceleração do aprendizado por reforço., $\mathrm{PhD}$ thesis, Universidade de São Paulo. https : //doi .org/10.11606/T. 3. 2004. tde-28062005-191041.

Bodin, L. (1983). Routing and scheduling of vehicles and crews, the state of the art, Comput. Oper. Res. 10(2): 63211. https://doi.org/10.1016/0305-0548 (83)90030-8.

Boeing, D. H. A. et al. (2019). Ensinando um robô a julgar: pragmática, discricionariedade e vieses no uso de aprendizado de máquina no judiciário. Disponível em https : //repositorio.ufsc.br/bitstream/handle/123456789/ 203514/TCC-Ensinandoumrobôajulgar1-3-merged.pdf? sequence $=1 \&$ is $\mathrm{s}$ llowed $=y$.

Brazdil, P., Carrier, C. G., Soares, C. and Vilalta, R. (2008). Metalearning: Applications to data mining, Springer Science \& Business Media. https://doi.org/10.1007/ 978-3-540-73263-1.

Cai, H., Lin, J., Lin, Y., Liu, Z., Wang, K., Wang, T., Zhu, L. and Han, S. (2020). Automl for architecting efficient and specialized neural networks, IEEE Micro 40(1): 75-82. https://doi.org/10.1109/MM.2019.2953153.
Celiberto Jr, L. A. (2007). Aprendizado por reforço acelerado por heurísticas no domínio do futebol de robôs simulado, Master's thesis, Centro Universitário da FEI. Disponível em https://repositorio.fei.edu.br/bitstream/ FEI/437/1/fulltext.pdf.

Chen, S., Wu, J. and Chen, X. (2019). Deep reinforcement learning with model-based acceleration for hyperparameter optimization, 2019 IEEE 31st International Conference on Tools with Artificial Intelligence (ICTAI), pp. 170177. https://doi.org/10.1109/ICTAI.2019.00032.

Espindola, G. M. d. (2009). Uso de algoritmos genéticos no ajuste de parâmetros da segmentação de imagens, XIV Simpósio Brasileiro de Sensoriamento Remoto, pp. 6861-6868. Disponível em http://marte.sid.inpe.br/col/dpi.inpe.br/sbsr@80/ 2008/11.01.20.18/doc/6861-6868.pdf.

Faria, G. (2000). Explorando o potencial de algoritmos de aprendizado com reforço em robôs móveis, Master's thesis, Universidade de São Paulo. https ://doi .org/10.11606/ D. 55.2020.tde-19022020-091603.

Feitosa, R. Q., Costa, G. A. O. P., Fredrich, C. M. B., Camargo, F. F. and de Almeida, C. M. (2009). Uma avaliação de métodos genéticos para ajuste de parâmetros de segmentação, XIV Simpósio Brasileiro de Sensoriamento Remoto, pp. 6875-6882. Disponível em http://marte. sid.inpe.br/col/dpi.inpe.br/sbsr@80/2008/11.18.13. 07/doc/6875-6882.pdf?languagebutton=pt-BR.

Feurer, M., Klein, A., Eggensperger, K., Springenberg, J., Blum, M. and Hutter, F. (2015). Efficient and robust automated machine learning, in C. Cortes, N. D. Lawrence, D. D. Lee, M. Sugiyama and R. Garnett (eds), Advances in Neural Information Processing Systems 28, Curran Associates, Inc., pp. 2962-2970. Disponível em https://proceedings.neurips.cc/paper/2015/ file/11d0e6287202f ced83f79975ec59a3a6-Paper.pdf.

Gambardella, L. M. and Dorigo, M. (1995). Ant-q: A reinforcement learning approach to the traveling salesman problem, Proceedings of the Twelfth International Conference on Machine Learning pp. 252-260. https : //doi.org/10.1016/B978-1-55860-377-6.50039-6.

Gershman, S. J. (2016). Empirical priors for reinforcement learning models, Journal of Mathematical Psychology 71: 1-6. https://doi.org/10.1016/j.jmp.2016.01.006.

Goldbarg, M. C. and Luna, H. P. L. (2005). Otimização combinatória e programação linear: modelos e algoritmos, Elsevier.

Hutter, F., Hoos, H. and Leyton-Brown, K. (2014). An efficient approach for assessing hyperparameter importance, Proceedings of International Conference on Machine Learning 2014 (ICML 2014), pp. 754-762. "Disponível em http://proceedings.mlr.press/v32/hutter14.pdf.

Hutter, F., Kotthoff, L. and Vanschoren, J. (eds) (2018). Automated Machine Learning: Methods, Systems, Challenges, Springer. https://doi.org/10.1007/ 978-3-030-05318-5. 
Júnior, F. C. D. L., Neto, A. D. D. and De Melo, J. D. (2010). Hybrid metaheuristics using reinforcement learning applied to salesman traveling problem, Traveling Salesman Problem, Theory and Applications, IntechOpen. https://doi.org/10.5772/13343.

Lakshmi, E. S., Rao, M. N. and Sudhamani, M. (2020). Response surface methodology-artificial neural network based optimization and strain improvement of cellulase production by streptomyces sp., Bioscience Journal 36(4): 1390-1402. Disponível em https: //docs.bvsalud.org/biblioref/2021/02/1147303/ 48006-article-text-229344-1-10-20200527 .pdf.

Leotti, V. B., Birck, A. R. and Riboldi, J. (2005). Comparação dos testes de aderência à normalidade kolmogorovsmirnov, anderson-darling, cramer-von mises e shapiro-wilk por simulação, Anais do $11^{\circ}$ Simpósio de Estatística Aplicada à Experimentação Agronômica . Disponível em https://www.inf.ufsc.br/ vera.carmo/Testes_ de_Hipoteses/Testes_aderencia.pdf.

Liu, F. and Zeng, G. (2009). Study of genetic algorithm with reinforcement learning to solve the tsp, Expert Systems with Applications 36(3): 6995-7001. https://doi. org $/ 10.1016 / j$. eswa. 2008.08.026.

Makmal, A., Melnikov, A. A., Dunjko, V. and Briegel, H. J. (2016). Meta-learning within projective simulation, IEEE Access 4: 2110-2122. https ://doi.org/10.1109/ access . 2016. 2556579.

Mantovani, R. G., Horváth, T., Cerri, R., Vanschoren, J. and de Carvalho, A. C. (2016). Hyper-parameter tuning of a decision tree induction algorithm, 5th Brazilian Conference on Intelligent Systems (BRACIS), IEEE, pp. 37-42. https://doi.org/10.1109/BRACIS. 2016.018.

Mantovani, R. G., Rossi, A. L., Alcobaça, E., Vanschoren, J. and de Carvalho, A. C. (2019). A meta-learning recommender system for hyperparameter tuning: Predicting when tuning improves svm classifiers, Information Sciences 501: 193-221. https://doi.org/10.1016/j.ins . 2019.06.005.

Mantovani, R. G., Rossi, A. L., Vanschoren, J., Bischl, B. and Carvalho, A. C. (2015). To tune or not to tune: recommending when to adjust svm hyper-parameters via meta-learning, 2015 International Joint Conference on Neural Networks (IJCNN), IEEE, pp. 1-8. https: //doi . org/10.1109/IJCNN. 2015.7280644.

Martins, M. F. (2007). Aprendizado por reforço acelerado por heurísticas aplicado ao domínio do futebol de robôs, Master's thesis, Centro Universitário da FEI. Disponível em https://repositorio.fei.edu.br/bitstream/ FEI/417/2/fulltext.pdf.

Mitchell, T. M. (1997). Machine Learning, McGraw-hill New York.

Monard, M. C. and Baranauskas, J. A. (2003). Conceitos sobre aprendizado de máquina, Sistemas Inteligentes Fundamentos e Aplicações, 1 edn, Manole Ltda, Barueri-SP, pp. 39-56. Disponível em https://dcm.ffclrp.usp.br/ augusto/publications/ 2003-sistemas-inteligentes-cap4.pdf.
Myers, R. H., Montgomery, D. C. and Anderson-Cook, C. M. (2016). Response surface methodology: process and product optimization using designed experiments, John Wiley \& Sons.

Ottoni, A. L. C. (2016). Análise de sensibilidade dos parâmetros do aprendizado por reforço na solução do problema do caixeiro viajante, Master's thesis, Programa de PósGraduação em Engenharia Elétrica da associação ampla CEFET-MG e UFSJ. Disponível em https://drive . google. com/open? id=0B33h6pvItVsQYktld2d6alU5RHM.

Ottoni, A. L. C., Nepomuceno, E. G., Cordeiro, L. T., Lamperti, R. D. and Oliveira, M. (2015). Análise do desempenho do aprendizado por reforço na solução do problema do caixeiro viajante, XII SBAI-Simpósio Brasileiro de Automação Inteligente pp. 43-48. Disponível em http://swge.inf.br/SBAI2015/anais/017.pdf.

Ottoni, A. L. C., Nepomuceno, E. G. and de Oliveira, M. S. (2016). Aprendizado por reforço na solução do problema do caixeiro viajante assimétrico: Uma comparação entre os algoritmos q-learning e sarsa, XII Simpósio de Mecânica Computacional. Disponível em https://www.ufsj.edu.br/portal2-repositorio/File/ gcom/OttoniSIMMEC2016.pdf.

Ottoni, A. L. C., Nepomuceno, E. G. and de Oliveira, M. S. (2017). Análise do desempenho do aprendizado por reforço na solução do problema da mochila multidimensional, Revista Brasileira de Computação Aplicada 9(3): 5670. https://doi.org/10.5335/rbca.v9i3.6601.

Ottoni, A. L. C., Nepomuceno, E. G., de Oliveira, M. S. and de Oliveira, D. C. R. (2020). Tuning of reinforcement learning parameters applied to sop using the scott-knott method, Soft Computing 24(6): 4441-4453. https://doi.org/10.1007/s00500-019-04206-w.

Ottoni, A. L. C., Nepomuceno, E. G. and Oliveira, M. S. (2016). Análise de sensibilidade dos parâmetros do aprendizado por reforço na solução do problema do caixeiro viajante: modelagem via superfície de resposta, XXI Congresso Brasileiro de Automática, Vol. 21, pp. 513-518. Disponível em http://www. swge.inf.br/ PDF/CBA2016-0170_047213.PDF.

Ottoni, A. L. C., Nepomuceno, E. G. and Oliveira, M. S. (2019). Estimação de parâmetros do aprendizado por reforço para o problema de planejamento de rotas com reabastecimento, Simpósio Brasileiro de Automação Inteligente. https://doi.org/10.17648/sbai-2019-111113.

Ottoni, A. L. C., Nepomuceno, E. G. and Oliveira, M. S. d. (2018). A response surface model approach to parameter estimation of reinforcement learning for the travelling salesman problem, Journal of Control, Automation and Electrical Systems 29(3): 350-359. https ://doi . org/10. 1007/s40313-018-0374-y.

Ottoni, L. T. C., Ottoni, A. L. C., Oliveira, M. S. d. and Nepomuceno, E. G. (2020). Autorl-tsp: Sistema de aprendizado por reforço automatizado para o problema do caixeiro viajante, XXIII Congresso Brasileiro de Automática. https://doi.org/10.48011/asba.v2i1.1658. 
Pedro, O. R. (2013). Uma abordagem de busca tabu para o problema do caixeiro viajante com coleta de prêmios, Master's thesis, Universidade Federal de Minas Gerais. Disponível em https ://www.ppgee.ufmg.br/defesas/962M.PDF.

Pellegrini, J. and Wainer, J. (2007). Processos de decisão de markov: um tutorial, Revista de Informática Teórica e Aplicada 14(2): 133-179. https://doi.org/10.22456/ 2175-2745.5694.

Razali, N. M., Wah, Y. B. et al. (2011). Power comparisons of shapiro-wilk, kolmogorov-smirnov, lilliefors and anderson-darling tests, Journal of Statistical Modeling and Analytics 2(1): 21-33. Disponível em https: //www.nbi.dk/ petersen/Teaching/Stat2019/Power_ Comparisons_of_Shapiro-Wilk_Kolmogorov-Smirn.pdf.

Reinelt, G. (1991). Tsplib-a traveling salesman problem library, ORSA journal on computing 3(4): 376-384. https : //doi.org/10.1287/ijoc.3.4.376.

Reinelt, G. (1995). Tsplib95, University Heidelberg . Disponível em http://comopt.ifi.uni-heidelberg.de/ software/TSPLIB95/tsp95.pdf.

Riboldi, J. and Nascimento, L. d. C. S. C. d. (1994). Metodologia de superfície de resposta: uma abordagem introdutória, Cadernos de matemática e estatística. Série B, Trabalho de apoio didático. Porto Alegre. No. 25 (nov. 1994), 83f. . https://doi.org/10183/205091.

Rossi, A. L. D. (2009). Ajuste de parâmetros de técnicas de classificação por algoritmos bioinspirados, Master's thesis, Universidade de São Paulo. https ://doi .org/10. 11606/D.55.2009.tde-06052009-114528.

Rossi, R. G. (2015). Classificação automática de textos por meio de aprendizado de máquina baseado em redes, $\mathrm{PhD}$ thesis, Universidade de São Paulo. https ://doi .org/10. 11606/T.55.2016.tde-05042016-105648.

Russell, S. J. and Norvig, P. (2013). Inteligência artificial, Campus, 3st ed.

Santos, J. P. Q. d. (2009). Uma implementação paralela híbrida para o problema do caixeiro viajante usando algoritmos genéticos, grasp e aprendizagem por reforço, Master's thesis, Universidade Federal do Rio Grande do Norte. Disponível em https://repositorio.ufrn.br/ bitstream/123456789/15221/1/JoaoPQS.pdf.

Santos, J. P. Q. d. (2014). Estratégias de busca reativa utilizando aprendizagem por reforço e algoritmos de busca local, PhD thesis, UFRN. Disponível em https://repositorio.ufrn.br/bitstream/123456789/ 19393/1/JoaoPauloQueirozDosSantos_TESE.pdf.

Santos, J. P. Q. d., de Melo, J. D., Neto, A. D. D. and Aloise, D. (2014). Reactive search strategies using reinforcement learning, local search algorithms and variable neighborhood search, Expert Systems with Applications 41(10): 4939-4949. https://doi.org/10.1016/j.eswa. 2014.01 .040 .

Serra, M. R. G. (2004). Aplicações de aprendizagem por reforço em controle de tráfego veicular urbano, Master's thesis, Universidade Federal de Santa Catarina. Disponível em https://repositorio.ufsc.br/bitstream/handle/ 123456789/87535/206482.pdf? sequence=1\&isAllowed=y.

Silva, A. L. M. (2014). Algoritmo baseado em evolução diferencial para solução de problemas de otimização combinatória, Master's thesis, Universidade Federal de Minas Gerais. Disponível em https : //www .ppgee .ufmg.br/ defesas/1039M.PDF.

Stamoulis, D., Ding, R., Wang, D., Lymberopoulos, D., Priyantha, N. B., Liu, J. and Marculescu, D. (2020). Single-path mobile automl: Efficient convnet design and nas hyperparameter optimization, IEEE Journal of Selected Topics in Signal Processing pp. 609 - 622. https : //doi.org/10.1109/JSTSP. 2020.2971421.

Stange, R. L. (2011). Adaptatividade em aprendizagem de máquina: conceitos e estudo de caso., Master's thesis, Universidade de São Paulo. https://doi .org/10.11606/D.3. 2011.tde-02072012-175054.

Sun, R., Tatsumi, S. and Zhao, G. (2001). Multiagent reinforcement learning method with an improved ant colony system, 2001 IEEE International Conference on Systems, Man and Cybernetics. e-Systems and e-Man for Cybernetics in Cyberspace (Cat. No. 01CH37236), Vol. 3, IEEE, pp. 1612-1617. https://doi.org/10.1109/ICSMC. 2001. 973515.

Sutton, R. S. (1988). Learning to predict by the methods of temporal differences, Machine learning 3(1): 9-44. https://doi.org/10.1007/BF00115009.

Sutton, R. S. (1996). Generalization in reinforcement learning: Successful examples using sparse coarse coding, Advances in neural information processing systems, pp. 1038-1044. Disponível em https://proceedings . neurips.cc/paper/1995/file/ 8f1d43620bc6bb580df6e80b0dc05c48-Paper.pdf.

Sutton, R. S. and Barto, A. G. (2018). Reinforcement learning: An introduction, MIT press, 2nd ed.

Tsiakmaki, M., Kostopoulos, G., Kotsiantis, S. and Ragos, O. (2019). Implementing automl in educational data mining for prediction tasks, Applied Sciences 10(1). https://doi.org/10.3390/app10010090.

Tuggener, L., Amirian, M., Rombach, K., Lörwald, S., Varlet, A., Westermann, C. and Stadelmann, T. (2019). Automated machine learning in practice: State of the art and recent results, 20196 th Swiss Conference on Data Science (SDS), pp. 31-36. https://doi.org/10.1109/SDS.2019. 00-11.

Vitor, A. (2015). Uma proposta de algoritmo genético híbrido para o problema do caixeiro viajante, $\mathrm{PhD}$ thesis, Universidade Federal do Paraná. https ://doi .org/1884/41345.

Wang, H., Cui, Z., Sun, H., Rahnamayan, S. and Yang, X.S. (2017). Randomly attracted firefly algorithm with neighborhood search and dynamic parameter adjustment mechanism, Soft Computing 21(18): 5325-5339. https://doi.org/10.1007/s00500-016-2116-z.

Watkins, C. J. C. H. (1989). Learning from delayed rewards, PhD thesis, King's College. Disponível em http://www. cs.rhul.ac.uk/ chrisw/new_thesis.pdf. 
Watkins, C. J. and Dayan, P. (1992). Q-learning, Machine learning 8(3-4): 279-292. https://doi.org/10.1007/ BF00992698.

Zhao, J.-h., Li, F. and Zhang, X.-x. (2012). Parameter adjustment based on improved genetic algorithm for cognitive radio networks, The Journal of China Universities of Posts and Telecommunications 19(3): 22-26. https: //doi.org/10.1016/S1005-8885(11)60260-4. 